13,18

\title{
О природе красного сдвига $G$-пика раман-спектра в эпитаксиальном двумерном слое
}

\author{
(C) С.Ю. Давыдов ${ }^{1}$, О.В. Посредник ${ }^{2}$ \\ ${ }^{1}$ Физико-технический институт им. А.Ф. Иофрфе РАН, \\ Санкт-Петербург, Россия \\ ${ }^{2}$ Санкт-Петербургский государственный электротехнический университет (ЛЭТИ), \\ Санкт-Петербург, Россия \\ E-mail: Sergei_Davydov@mail.ru
}

Поступила в Редакцию 6 декабря 2020 г.

В окончательной редакции 6 декабря 2020 г.

Принята к публикации 7 декабря 2020 г.

В модели, исключающей наличие деформации на контакте монослоя и подложки, рассмотрена роль межплоскостного упругого взаимодействия. Показано, что это взаимодействие приводит к увеличению значений фононных частот. Кратко обсуждается роль внешнего давления и температуры.

Ключевые слова: силовые константы, фононы, давление, температура.

DOI: 10.21883/FTT.2021.04.50723.253

\section{1. Введение}

В настоящее время среди экспериментаторов популярно считать причиной красного сдвига рамановского $G$-пика $\Delta \omega_{G}$ эпитаксиального графена (эпиграфена) наличием упругой деформации на интерфейсе, возникающей вследствие различия постоянных решеток пленки и подложки. Впервые, насколько известно авторам, подобное объяснение сдвига было предложено в работе [1] при анализе рамановского спектра аморфного графитового слоя, сформированного на кремниевой пластине. Такая схема возникла благодаря давно уже установленной для алмазоподобных кристаллов зависимости $\Delta \omega_{G} \propto \sigma$, где $\sigma$ - внешнее одноосное механическое напряжение [2] (та же зависимость имеет место и для свободного графена [3]). По нашему мнению, однако, первопричиной красного смещения $G$-пика эпитаксиального слоя является само взаимодействие слой-подложка, независимо от того, появляется ли при этом на интерфейсе напряжение или нет. Справедливость такого утверждения была продемонстрирована в работе [4] в рамках модели двух связанных осцилляторов. Здесь мы рассмотрим задачу о сдвиге $G$-пика в рамках более сложной модели эпитаксиального монослоя и субстрата, изначально исключающей деформацию на интерфейсе.

\section{2. Модель эпитаксиальной структуры}

Предлагаемая модель (рис. 1) представляет собой двумерную (2D) квадратную решетку, расположенную в плоскости $(x, y, 0)$ (в дальнейшем - монослой $(\mathrm{ML}))$, расположенную на поверхности (100) полубесконечной простой кубической решетки (SISCL). Обе решетки являются двухатомными структурами с массами составляющих их атомов $m_{1,2}(\mathrm{ML})$ и $M_{1,2}$ (SISCL) и одинако- вым расстоянием между ближайшими соседями, равным $a$, что исключает наличие механических напряжений на интерфейсе. Расстояние между ML и SISCL положим равным $a^{*}$. В дальнейшем будем рассматривать только плоские (in-plane) колебания, характеризуемые смещениями $u_{i, j}$ атома $i$ в плоскости $j=0,-1,-2, \ldots$ в направлении оси $x$. Для описания взаимодействия ближайших соседей (б.с.) введем центральные (bondstretching) силовые константы $\alpha$ и $\beta$ для ML и SISCL coответственно. Взаимодействие между б. с. ML и поверхностью (100) SISCL описываем нецентральной (bondbending) силовой константой $\gamma^{*}$; межплоскостное взаимодействие между б.с., принадлежащими к внутренним плоскостям SISCL характеризуем нецентральной

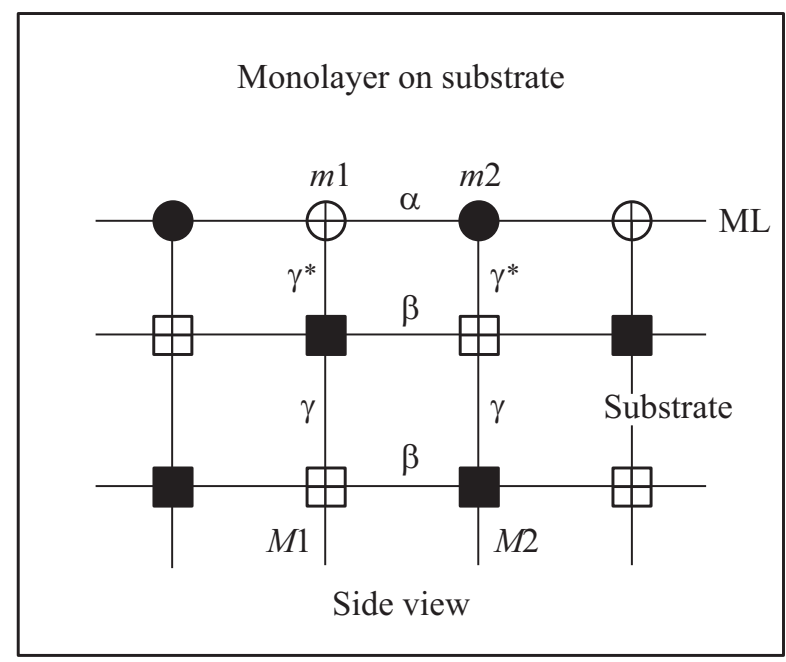

Рис. 1. Структурная модель двумерного эпитаксиального слоя, в котором отсутствуют механические напряжения на интерфейсе. 
силовой константой $\gamma$ (рисунок). Тогда для смещений $u_{i j}=U_{i j} \exp \left[i\left(k_{x} x_{i}+k_{z} z_{j}\right)\right]$, где $U_{i j}-$ амплитуды колебаний, $k_{x}-$ волновой вектор $\left(\left|k_{x}\right| \leq \pi / 2 a\right), k_{z}-$ действительное число, уравнения движения имеют вид

$$
\begin{gathered}
m_{1} \omega^{2} U_{00}=\left(2 \alpha+\gamma^{*}\right) U_{00}-2 \alpha \cos \left(k_{x} a\right) U_{10}-\gamma^{*} U_{0-1} e^{-i k_{z} a^{*}}, \\
m_{2} \omega^{2} U_{10}=\left(2 \alpha+\gamma^{*}\right) U_{10}-2 \alpha \cos \left(k_{x} a\right) U_{00}-\gamma^{*} U_{1-1} e^{-i k_{z} a^{*}}, \\
M_{2} \omega^{2} U_{0-1}=\left(2 \beta+\gamma+\gamma^{*}\right) U_{0-1}-2 \beta \cos \left(k_{x} a\right) U_{-1-1} \\
-\gamma^{*} U_{00} e^{i k_{z} a^{*}}-\gamma U_{0-2} e^{-i k_{z} a}, \\
M_{1} \omega^{2} U_{1-1}=\left(2 \beta+\gamma+\gamma^{*}\right) U_{1-1}-2 \beta \cos \left(k_{x} a\right) U_{0-1} \\
-\gamma^{*} U_{10} e^{i k_{z} a^{*}}-\gamma U_{1-2} e^{-i r_{z} a}, \\
M_{2} \omega^{2} U_{0-2}= \\
2(\beta+\gamma) U_{0-2}-2 \beta \cos \left(k_{z} a\right) U_{1-2} \\
-\gamma\left(U_{0-1} e^{i k_{z} a^{*}}+U_{0-3} e^{-i k_{z} a}\right),
\end{gathered}
$$$$
M_{1} \omega^{2} U_{1-2}=2(\beta+\gamma) U_{1-2}-2 \beta \cos \left(k_{x} a\right) U_{0-2}
$$$$
-\gamma\left(U_{1-1} e^{i k_{z} a}+U_{1-3} e^{-i k_{z} a}\right)
$$

и т.д. Так как нас в первую очередь интересует воздействие подложки на ML, рассмотрим для начала двухслойную структуру. Легко показать, что фононный спектр такой системы определяется из уравнения $D=0$, где

$$
\begin{aligned}
D= & \left(A_{1} A_{2}-a^{2}\right)\left(B_{1} B_{2}-b^{2}\right) \\
& -\left(\gamma^{*}\right)^{2}\left(A_{1} B_{1}+A_{2} B_{2}+2 a b\right)+\left(\gamma^{*}\right)^{4},
\end{aligned}
$$

где $A_{1,2}=m_{1,2} \omega^{2}-2 \alpha-\gamma^{*}, \quad B_{1,2}=M_{1,2} \omega^{2}-2 \beta-\gamma^{*}$, $a=2 \alpha \cos \left(k_{x}, a\right), b=2 \beta \cos \left(k_{x} a\right)$. Для свободного ML и бесконечной простой кубической решетки (ISCL) имеем соответственно

$$
\begin{gathered}
\omega_{\mathrm{ML}}^{2}=\alpha \bar{m}^{-1}\left(1 \pm \sqrt{1-\left(4 \bar{m}^{2} / m_{1} m_{2}\right) \sin ^{2}\left(k_{x} a\right)}\right) \\
\bar{m}^{-1}=m_{1}^{-1}+m_{2}^{-1}, \\
\omega_{\mathrm{ISCL}}^{2}=(\beta+\gamma) \bar{M}^{-1}\left(1 \pm \sqrt{1-\left(4 \bar{M}^{2} / M_{1} M_{2}\right) \sin ^{2}\left(k_{x} a\right)}\right), \\
\bar{M}^{-1}=M_{1}^{-1}+M_{2}^{-1} .
\end{gathered}
$$

Частотный спектр SISCL со свободной поверхностью приведен в [5].

Положим для простоты $\gamma^{*} \ll \alpha, \beta, \omega=\omega_{A(B)}+v_{A(B)}$, $\left|v_{A(B)}\right| \ll \omega_{A(B)}$, где индексы $A$ и $B$ относятся к верхней (ML) и нижней плоскостям двухслойной структуры. Тогда из (2) в линейном по $\gamma^{*}$ приближении для ML получим

$$
\begin{gathered}
\omega_{A}^{2}=\alpha \bar{m}^{-1}\left(1 \pm \sqrt{1-\left(4 \bar{m}^{2} / m_{1} m_{2}\right) \sin ^{2}\left(k_{x} a\right)}\right) \\
\bar{m}^{-1}=m_{1}^{-1}+m_{2}^{-1}
\end{gathered}
$$

$$
v_{A}=\gamma^{*} \frac{\left(m_{1}+m_{2}\right) \omega_{A}^{2}-4 \alpha}{4 \omega_{A}\left[m_{1} m_{2} \omega_{A}^{2}-\alpha\left(m_{1}+m_{2}\right)\right]} .
$$

Для частотных характеристик в центре зоны Бриллюэна $\left(k_{z}=0\right)$ получаем

$$
\begin{gathered}
\omega_{A}^{o p t}(0)=\sqrt{2 \alpha / \bar{m}}, \\
v_{A}^{o p t}(0)=\gamma^{*} \frac{m_{1}^{2}+m_{2}^{2}}{2 m_{1} m_{2}\left(m_{1}+m_{2}\right)} \frac{1}{\omega_{A}^{o p t}(0)}, \quad v_{A}^{a c}(0)=0,
\end{gathered}
$$

где верхние индексы $o p t$ и $a c$ обозначают оптические и акустические моды. Подчеркнем, что именно значение $v_{A}^{\text {opt }}(0)$ характеризует сдвиг $G$-пика раман-спектра $\mathrm{ML}$, вызванный взаимодействием с SISCL. Здесь удобно ввести безразмерное отношение $\eta_{A(B)}=v_{A(B)} / \omega_{A(B)}$, где $\omega_{A(B)} \neq 0$, так что $\omega=\omega_{A(B)}\left(1+\eta_{A(B)}\right)$. Из (7) находим

$$
\eta_{A}^{o p t}(0)=\left(\gamma^{*} / 4 \alpha\right) \frac{m_{1}^{2}+m_{2}^{2}}{\left(m_{1}+m_{2}\right)^{2}} .
$$

Таким образом, относительный сдвиг $G$-пика в первом приближении пропорционален отношению $\gamma^{*} / \alpha$, что совпадает с результатом работы [4], где силовым константам $\alpha$ и $\gamma^{*}$ соответствуют силовые константы $k_{0 g}$ и $k$. Полагая $m_{1} \sim m_{2}$ и $\gamma^{*} / \alpha=0.1$, получим $\eta_{A}^{\text {opt }}(0) \sim 10^{-2}$, что отлично согласуется с экспериментом для графена на карбиде кремния [4]. Принимая $m_{1}=m+\Delta m \quad$ и $m_{2}=m-\Delta m$, получим $\eta_{A}^{o p t}(0)=\left(\gamma^{*} / 8 \alpha\right)\left(1+\Delta m^{2}\right)$. Для акустической ветви спектра на границе зоны Бриллюэна $\left(k_{x}=\pi / 2 a\right)$ при $m_{1}>m_{2}$ имеем

$$
\begin{gathered}
\omega_{A}^{a c}(\pi / 2 a)=\sqrt{2 \alpha / m_{1}}, \quad v_{A}^{a c}(\pi / 2 a)=\gamma^{*} / 2 \sqrt{2 \alpha / m_{1}}, \\
\eta_{A}^{a c}(\pi / 2 a)=\gamma^{*} / 4 \alpha .
\end{gathered}
$$

Выражения, аналогичные (5)-(9), можно записать для плоскости $B$, заменяя $\alpha$ на $\beta$ и $m_{1,2}$ на $M_{1,2}$. Таким образом, учет взаимодействия между плоскостями ведет к увеличению значений всех частот (за исключением $\left.\omega^{a c}(0)=0\right)$. Легко показать, что дополнительно полагая $\gamma \ll \beta$ (слоистая структура) и имея в виду дальнейшую линеаризацию по параметрам $\gamma^{*}$ и $\gamma$, для определения дисперсии фононов можно вместо (2) записать выражение $D_{\text {sub }}^{\text {surf }} \approx\left(M_{1} \omega^{2}-2 \beta-\gamma-\gamma^{*}\right)\left(M_{2} \omega^{3}-2 \beta-\gamma-\gamma^{*}\right)-b^{2}$ для плоскости (100) и $D_{\text {sub }}^{\text {bulk }} \approx\left(M_{1} \omega^{2}-2(\beta+\gamma)\right.$ $\times\left(M_{2} \omega^{3}-(2 \beta+\gamma)\right)-b^{2}$ для внутренних плоскостей SISCL. Заменяя в формулах (5)-(9) массы $m_{1,2}$ на $M_{1,2}$ и силовую константу $\alpha$ соответственно на $\beta+\gamma / 2$ и $\beta+\gamma$, получим выражения для частотных характеристик.

\section{3. Влияние давления и температуры на сдвиг $G$-пика}

До сих пор мы пользовались гармоническим приближением, сейчас же перейдем к оценкам ангармонических характеристик фононного спектра ML. Отмеченное 
выше соответствие между отношениями $\gamma^{*} / \alpha$ и $k / k_{0 g}$ позволяет использовать выводы [4] о зависимости сдвига $G$-пика от давления $P$ и температуры $T$ для качественных оценок. Мы, однако, рассмотрим зависимости $v_{A(B)}(P)$ и $v_{A(B)}(T)$ более подробно, воспользовавшись результатами работ $[6,7]$, но пренебрегая, для простоты, возможной полярностью связей. Так как для ковалентных $\sigma$ - и $\pi$-связей, образованных $\mathrm{sp}^{k}$-орбиталями $(k=1,2,3)$, силовые константы пропорциональны $a^{-4}$ или $\left(a^{*}\right)^{-4}[6-8]$, то при деформации в линейном приближении имеем $\widetilde{\alpha} \sim \alpha \vartheta, \widetilde{\beta} \sim \beta \vartheta, \widetilde{\gamma} \sim \gamma \vartheta$ и $\widetilde{\gamma}^{*} \sim \gamma^{*} \vartheta^{*}$, где $\vartheta=1 \mp 4 \Delta a / a, \vartheta^{*}=1 \mp 4 \Delta a^{*} / a^{*}$, верхний знак отвечает сжатию $(\Delta a<0)$, нижний $(\Delta a>0)$ - растяжению и $\Delta a / a \propto \Delta P / B(B-$ модуль сжатия $)$. Вводя обозначения $\omega_{A}^{o p t}(0)=\omega_{G A}$ и $v_{A}^{o p t}(0)=v_{G A}$, получим $\omega_{G A}^{\prime} \sim \omega_{G A}(1 \mp 2 \Delta a / a)$ и $\widetilde{v}_{G A} \sim v_{G A}(1 \mp 2 \Delta a / a)$, причем аналогичные формулы справедливы для $\omega_{G B}^{\prime}$ и $v_{G B}^{\prime}$. При оценке $v_{G A}^{\prime}$ учтено, что в линейном приближении следует положить $\Delta a^{*} / a^{*}=\Delta a / a$. Здесь мы игнорировали появление на интерфейсе упругих напряжений при $\alpha \neq \beta$ как вторичный эффект, наведенный внешним давлением.

Сдвиг $G$-пика под действием внешнего давления в углеродных (графеноподобных) структурах экспериментально исследовался в работах $[9,10]$, где в диапазоне изменений $P$ от 0 до $\sim 5-10 \mathrm{GPa}$ наблюдался практически линейный рост $\omega_{G B}^{\prime}$. Более того, в [9] при постоянной Грюнайзена $\bar{\gamma} \approx 2$ (что соответствует результатам [6,7]) для одноосной деформации получено совпадающее с нашим отношение $\Delta \omega_{G} / \omega_{G} \approx 2|\Delta a| / a$.

Перейдем теперь к оценкам влияния температуры на $G$-пик ML. При этом вновь воспользуемся результатами работ $[6,7]$, представив квазигармоническую свободную энергию ML (на связь) в виде $F=U_{e l}+F_{v i b}$, где $U_{e l}-$ упругая энергия, $F_{v i b}-$ колебательная энергия. При температурах выше температуры Дебая $T_{D}$ положим $F_{v i b} \sim(3 T / N) \ln \left(\omega_{G A}^{\prime \prime} / T\right)$, где $N=2,3$ - размерность структуры, температура $T$ и частота $\omega_{G}^{\prime \prime}$ измеряются в энергетических единицах. Тогда зависящая от температуры силовая константа $\alpha^{\prime \prime}=\alpha_{e l}^{\prime \prime}+\alpha_{v i b}^{\prime \prime}$. Легко показать, что упругая составляющая $\alpha_{e l}^{\prime \prime} \sim \alpha\left(1-\alpha_{T} T\right)$, где $\alpha_{T}$ - коэффициент линейного теплового расширения (оценки $[6,7]$ дают $\alpha_{T} \sim(5-8) \cdot 10^{-6} \mathrm{~K}^{-1}$. Согласно [11], $\alpha_{T} \sim 6.5 \cdot 10^{-6} \mathrm{~K}^{-1}$ для свободного графена и $\alpha_{T} \sim 8.7 \cdot 10^{-6} \mathrm{~K}^{-1}$ для слабосвязанного эпитаксиального графена на поверхности карбида кремния, что, в рамках нашей модели, объясняется наличием связи ML c SISCL.

Для колебательной составляющей центральной силовой константы получим $\alpha_{v i b}^{\prime \prime} \sim\left(3 T / 2 N\left(\omega_{G}^{\prime \prime}\right)^{2}\right)$ $\times\left[\partial^{2}\left(\omega_{2}^{\prime \prime}\right)^{2} / \partial a^{2}-\left(\partial\left(\omega_{G}^{\prime \prime}\right)^{2} / \partial a\right)^{2} /\left(\omega_{G}^{\prime \prime}\right)^{2}\right]$. Так как $\left(\partial \ln \alpha^{\prime \prime} / \partial T\right) \propto(\partial \ln B / \partial T)$, оценки [6,7] для температурной зависимости модуля сжатия показывают, что знаки производных $\partial \alpha_{e l}^{\prime \prime} / \partial T$ и $\partial \alpha_{v i b}^{\prime \prime} / \partial T$ одинаковы, откуда имеем $\partial \alpha_{v i b}^{\prime \prime} / \partial T<0$. Далее, в рамках самосогласованного квазигармонического приближения [12] температура уменьшает межплоскостные силовые константы $\gamma^{*}$ и $\gamma$ как в трехмерных кристаллах [13], так и в наноструктурах [14]. Отсюда следует, что $\partial \omega_{G}^{\prime \prime} / \partial T<0$. Здесь мы не учитываем возникновение на интерфейсе термоупругих напряжений, вызванных разницей коэффициентов теплового расширения вследствие различия силовых констант $\alpha$ и $\beta$, как вторичный эффект, наведенный температурой.

Температурный сдвиг $G$-пика экспериментально исследовался для графена в [15-18] и h-BN в [18], где, во-первых, показано, что энергия пика с ростом $T$ линейно уменьшается: для графена $\partial \omega_{G}^{\prime \prime} / \partial T \approx-(0.015-0.020) \mathrm{cm}^{-1} \cdot \mathrm{K}^{-1}, \quad$ для $\mathrm{h}-\mathrm{BN}$ $\partial \omega_{G}^{\prime \prime} / \partial T \approx-0.03 \mathrm{~cm}^{-1} \cdot \mathrm{K}^{-1}$. Во-вторых, согласно [16], для графена, сформированного на $6 \mathrm{H}-\mathrm{SiC}(0001)$, температурный сдвиг в 4 раза больше, чем у свободного графена, что, на наш взгляд, свидетельствует о роли связи ML-SISCL. Аналогичные эффекты наблюдаются для подложек $\mathrm{SiO}_{2} / \mathrm{Si}, \mathrm{Au} / \mathrm{SiN}_{x} / \mathrm{Si}, \mathrm{Cu}[16,17]$.

\section{4. Заключение}

Итак, в настоящей работе с помощью простой модели и полуколичественных оценок мы продемонстрировали, что связь эпитаксиального слоя с подложкой ведет к красному смещению $G$-пика $\Delta \omega_{G}$, усиливает рост $\Delta \omega_{G}$ с увеличением внешнего давления и спад $\Delta \omega_{G}$ с ростом температуры. Выявленные тенденции в целом согласуются с известными авторам экспериментальными данными, хотя относительно $P$ - и $T$-зависимостей $\Delta \omega_{G}$ требуются дополнительные исследования.

Деформационное описание $\Delta \omega_{G}$ для углеродных наноструктур стало популярным после появления работ $[19,20]$, в которых полагали $\Delta \omega_{G}=C \sigma$, где $\sigma-$ механическое напряжение, а коэффициент $C=7.47 \mathrm{~cm}^{-1} \cdot \mathrm{GPa}^{-1}$. В дальнейшем коэффициент $C$ стал рассматриваться как универсальный, а из экспериментального значения $\Delta \omega_{G}$ определяли величину $\sigma$. Тут сразу же возникают два возражения. Во-первых, представляется странным, что графен, обладающий минимальной постоянной решетки, испытывает сжатие $(\sigma>0)$ со стороны подложки (на эту странность указывалось еще в работе [21]). Во-вторых, не ясно, как вызываемая подложкой деформация узлов большой элементарной ячейки эпиграфена (см., например, соответствующие рисунки в [20] и [22]), которая, очевидно, является переменной как по величине, так и по знаку, может быть описана в приближении $\sigma=$ const. Поэтому мы предлагаем анализировать измеренное отношение $\Delta \omega_{G} / \omega_{G}$ по формуле (8), извлекая из эксперимента значения отношения $\gamma^{*} / \alpha$.

\section{Конфликт интересов}

Авторы заявляют об отсутствии конфликта интересов. 


\section{Список литературы}

[1] J.W. Ager III, S. Anders, A. Anders, I.G. Brown. Appl. Phys. Lett. 66, 3444 (1995).

[2] E. Anastassakis, A. Pinczuk, E. Burstein, F.H. Pollak, M. Cardona. Solid State Commun. 8, 133 (1970).

[3] K.L. Yang, J.O. Lee, H. Choo, F. Yang. J. Chem. Phys. C 122, 24467 (2018).

[4] С.Ю. Давыдов. ФТТ 59, 610 (2017).

[5] S. Takeno. Prog. Theor. Phys. 30, 1 (1963).

[6] С.Ю. Давыдов. Письма в ЖТФ 37, 24, 42 (2011).

[7] С.Ю. Давыдов, О.В. Посредник. ФТТ 15, 819 (2015).

[8] У. Харрисон. Электронная структура и свойства твердых тел. Мир, М. (1983).

[9] J.E. Proctor, E. Gregoryanz, K.S. Novoselov, M. Lotya, J.N. Coleman, M.P. Halsall. Phys. Rev. B 80, 073408 (2009).

[10] S. Lu, M. Yao, X. Yang, Q. Li, J. Xiao, Z. Yao, L. Jiang, R. Liu, B. Liu, S. Chen, B. Zou, T. Cui, B. Liu. Chem. Phys. Lett. 585, 101 (2013).

[11] M. Pazzo, D. Alfe, P. Lacovig, P. Hofmann, S. Lizzit, A. Baraldi. Phys. Rev. Lett. 106, 135501 (2011).

[12] P.B. Allen. Modern Phys. Lett. B 34, 2050025 (2020).

[13] L. Pietronero, E. Tosatti. Solid State Commun. 32, 255 (1979).

[14] С.Ю. Давыдов. ФТТ ]bf57, 1437 (2015).

[15] I. Calizo, A.A. Balandin, W. Bao, F. Miao, C N. Lau. Nano Lett. 7, 2645 (2007).

[16] N. Ferralis, R. Maboudian, C. Carraro. Phys. Rev. B 83, 081410(R) (2011).

[17] M.S. Tivanov, E.A. Kolesov, A.G. Praneuski, O.V. Korolik, A.M. Saad, I.V. Komissarov, N.G. Kovalchuk. J. Mater. Sci. Matter Electron 27, 8879 (2016).

[18] X. Li, J. Liu, K. Ding, X. Zhao, S. Li, W. Zhou, B. Liang. Nanoscale Res. Lett. 13, 25 (2018).

[19] H. Sakata, G. Dresselhaus, M.S. Dresselhaus, M. Enda. J. Appl. Phys. 63, 2769 (1988)

[20] Z.H. Ni, W. Chen, X.F. Fan, J.L. Kuo, T. Yu, A.T.S. Wee, Z.X. Shen. Phys. Rev. B 77, 115416 (2008).

[21] J. Röhrl, M. Hundhausen, K.V. Emtsev, T. Seyller, R. Graupner, L. Ley. Appl. Phys. Lett. 92, 201918 (2008).

[22] K.V. Emtsev, F. Speck, Th. Seyller, L. Ley, J.D. Riley. Phys. Rev. B 77, 155303 (2008).

Редактор Т.Н. Василевская 